\title{
Canine Cyclic Hematopoiesis is Associated with Abnormal Purine and Pyrimidine Metabolism
}

\author{
W. R. A. Osborne, W. P. Hammond, and D. C. Dale, Departments of Pediatrics \\ and Medicine, University of Washington School of Medicine, \\ Seattle, Washington 98195
}

\begin{abstract}
A B S T R A C T Canine cyclic hematopoiesis is an autosomal recessive disease characterized by regular 1113-d cycles of the neutrophil, reticulocyte, and platelet counts caused by a defect in regulation of marrow stem cell proliferation. Treatment with lithium abrogates cycling of the cell counts in these grey collie dogs. Aware of the defective lymphopoiesis associated with adenosine deaminase and purine nucleoside phosphorylase deficiencies, we hypothesized that abnormal purine or pyrimidine metabolism might be present in these dogs. Using high pressure liquid chromatography, we measured erythrocyte purine and pyrimidine nucleotide levels and plasma purine and pyrimidine nucleosides and bases in normal and grey collie dogs before and during lithium treatment.

During neutropenic periods in the grey collies, erythrocyte ATP, GTP, and UTP levels were significantly elevated. Normal dogs made neutropenic with cyclophosphamide did not show such elevations. Lithium treatment normalized the levels of erythrocyte ATP, GTP, and UTP in the grey collies and eliminated the differences between normal and grey collie nucleotide levels. Plasma thymine levels were markedly increased during neutropenia in the grey collie but were not increased in cyclophosphamide-treated normal dogs. The finding of abnormal concentrations of purine and pyrimidine metabolites in these dogs suggest that a metabolic derangement in purine or pyrimidine metabolism may be the cause of the defective stem cell proliferation in this disease.
\end{abstract}

\section{INTRODUCTION}

Cyclic hematopoiesis in grey collie dogs is an autosomal recessive disorder in which regular 11-13-d cycles of the neutrophil count lead to severe infections

Address reprint requests to Dr. Osborne.

Received for publication 21 July 1982 and in revised form 19 January 1983. and early death. The cyclic fluctuation of the neutrophil count is reproducible and is reliably associated with fluctuations in eosinophils, monocytes, lymphocytes, platelets, and reticulocytes (1-4). These periodic changes are caused by alterations in marrow cell production rates rather than alterations in rates of destruction or sequestration of cells in the circulation $(2,4-6)$. Studies of granulocytic and erythroid precursor cells have shown fluctuations in these committed progenitor cell populations over the cycle of cell counts but have not explained the mechanism(s) causing cyclic hematopoiesis (7-11). Bone marrow transplantation experiments have shown that this disease can be cured or transferred to a normal littermate by irradiation and marrow cell infusion, clearly implicating the marrow stem cell pool(s) as the site of the defect (12-14). Recently we have demonstrated that lithium carbonate eliminates the severe recurrent neutropenia, smooths the fluctuations of other cell counts, and eliminates the marked cycling of neutrophilic colony-forming cells in these dogs $(15,16)$.

In autosomal recessive disorders where the biochemical defect has been identified, it usually involves an enzyme protein. Drawing an analogy with the defective lymphopoiesis associated with adenosine deaminase (ADA) $)^{1}(17)$ and purine nucleoside phosphorylase (NP) (18) deficiency, we reasoned that a similar phenomenon of abnormal purine metabolism might be present in cyclic hematopoiesis. Studies of patients with inherited immune deficiency have shown that the absence of ADA is associated with combined T and B lymphocyte dysfunction and the absence of NP is associated with a severe $\mathrm{T}$ cell defect and normal B cell function (19). It is generally accepted that the accumulation of dATP in ADA deficiency and deoxy-

\footnotetext{
${ }^{1}$ Abbreviations used in this paper: ADA, adenosine deaminase; GC, grey collie; HGPRT, hypoxanthine guanine phosphorylase transferase; ND, normal dog; NP, purine nucleoside phosphorylase; PMN, neutrophils.
} 
guanosine triphosphate (dGTP) in NP deficiency contribute to the immune defects (20-22). It is postulated that their accumulation inhibits ribonucleotide reductase, an enzyme central to the production of deoxynucleotide triphosphate precursors for DNA synthesis (23).

These studies have emphasized that rapidly dividing lymphocytes are uniquely susceptible to defects in purine and pyrimidine metabolism that cause an imbalance in the supply of precursors for DNA synthesis. Because erythrocytes are technically easy to obtain, they have been extensively studied in our laboratory and are known to act as passive traps for circulating purines and pyrimidines that become phosphorylated in ADA and NP deficiency, we hypothesized that erythrocyte nucleotide levels would reflect such a defect. As an initial search for this kind of metabolic defect in cyclic hematopoiesis, we have therefore examined erythrocyte purine and pyrimidine nucleotide concentrations and plasma purine and pyrimidine nucleosides and bases in grey collie and normal dogs prior to and during lithium treatment.

\section{METHODS}

Dogs. Normal and grey collie dogs were housed and cared for in the animal care facility of the University of Washington as previously described $(10,11)$. Blood specimens were obtained from the cephalic veins of unanesthetized dogs between 7:30 and 9:30 a.m. Blood cell counts on EDTAanticoagulated aliquots were performed by standard laboratory methods (15). Blood specimens for nucleotide and nucleoside studies were collected in heparinized syringes and the plasma and erythrocytes were separated by centrifugation at $600 \mathrm{~g}$ for $10 \mathrm{~min}$. The packed erythrocytes were lysed $1: 1$ with water and the hemoglobin determined (24). Proteinfree extracts of plasma and hemolysates were prepared by the method of Garrett and Santi (25) in 20 min and no later than $40 \mathrm{~min}$ from the time of withdrawal. Erythrocyte nucleotides were standardized using the mean hemolysate hemoglobin.

Dog treatment. For studies of erythrocytes from an animal with an elevated reticulocyte count, a normal dog was phlebotomized $20 \%$ of its blood volume every other day for $8 \mathrm{~d}$ to reduce its hematocrit by $20 \%$ and increase its reticulocyte count fivefold. For studies of drug-induced neutropenia, three normal dogs were given cyclophosphamide $(20$ $\mathrm{mg} / \mathrm{kg}, \mathrm{i} . \mathrm{v}$.) and followed through the predictable periods of neutropenia and rebound neutrophilia. For studies of lithium carbonate therapy, both normal and grey collie dogs were given the drug initially at $300 \mathrm{mg}$ q.i.d.; the dosage was then adjusted to maintain a serum lithium level between 0.8 and $1.25 \mathrm{meq} /$ liter, as described previously $(15,16)$. During each treatment period serial specimens for blood counts as well as plasma and erythrocyte nucleoside/nucleotide studies were collected as described above.

Cycle analysis. For comparisons of data from several grey collie dogs, the first day that the neutrophil count fell below $1,000 / \mathrm{mm}^{3}$ was defined as cycle day 1 ; each day was then numbered sequentially until the next occurrence of a cycle day 1 . Severe neutropenia usually lasted 4 to $6 \mathrm{~d}$, was fol- lowed by rebound to normal or elevated levels that lasted 6-8 d, and then was followed by recurrent neutropenia.

Erythrocyte nucleotide analysis. Purine and pyrimidine nucleotides were analyzed using a Waters (Waters Associates, Inc., Milford, MA) high-pressure liquid chromatograph equipped with a dual pump system, a programable gradient maker and an ultraviolet detector set at $254 \mathrm{~nm}$ and 0.1 fullscale absorbance coupled to a Texas Instrument chart recorder (Texas Instruments, Inc., Dallas, TX) and an Autolab integrator (Spectra-Physics, San Jose, CA). The column used was a strong anion exchanger supplied by Whatman, Inc., Clifton, NJ, with dimensions $4.6 \mathrm{~mm} \times 25 \mathrm{~cm}$. The nucleotides were eluted by gradient elution with $7 \mathrm{mM} \mathrm{KH}_{2} \mathrm{PO}_{4}$ low strength buffer and $0.25 \mathrm{M} \mathrm{KH}_{2} \mathrm{PO}_{4} / 0.5 \mathrm{M} \mathrm{KCl}$ high strength buffer, $\mathrm{pH} 3.5$, based on a modification of the method of Hartwick and Brown (26). A nonlinear gradient was used over a $30-\mathrm{min}$ period, such that the first half of the gradient was predominantly at low ionic strength followed by an exponentially increasing buffer molarity. The total nucleotide elution required $\sim 80 \mathrm{~min}$. An extract derived from $60 \mu \mathrm{l}$ of packed erythrocytes was analyzed.

The identification of peaks was by comparison with the retention times of known standards, and confirmed by coelution when known nucleotides were added to cell extracts. Because of the high resolution and extreme reproducibility of the separations, peak identification was unambiguous. The nucleotides were quantified by comparing peak areas to those obtained from known standards. The peak areas were obtained from the Autolab integrator, and were confirmed using the formula peak height $\times$ peak width at half height.

Plasma nucleoside and base analysis. The purine and pyrimidine nucleosides and bases were determined in plasma extracts by high-pressure liquid chromatography using a Waters reverse-phase $C_{18}$ column (27). The nucleosides and bases were eluted with a linear gradient made from $10 \mathrm{mM}$ $\mathrm{KH}_{2} \mathrm{PO}_{4} \mathrm{pH} 5.3$ and $80 \%$ methanol $/ \mathrm{H}_{2} \mathrm{O}$. The linear gradient was for a $30-\mathrm{min}$ period with $0-25 \%$ of the $80 \%$ methanol solution at a flow rate of $1.5 \mathrm{ml} / \mathrm{min}$ with the UV detector set at $254 \mathrm{~nm}$ and 0.05 absorbance units full-scale. The column output was also passed through a variable wavelength detector set at $280 \mathrm{~nm} 0.04$ full-scale absorbance and the signals from the detectors were recorded on a dual pen Texas Instruments chart recorder. The comparison between the two wavelengths helped to differentiate the mixture of purines and pyrimidines. Peaks were identified by retention time and coelution with known standards. The peaks were quantitated using an Autolab integrator using values derived from known standards.

Enzyme analysis. Separated and washed erythrocyte and leukocyte lysates were analyzed for ADA (28) and purine NP as previously described (29).

\section{RESULTS}

Erythrocyte nucleotide levels in untreated grey collies and normal dogs. Initial studies of three normal collies and four grey collies showed striking differences in nucleotide profiles between normal collies and grey collies with zero neutrophil count. The chromatograms demonstrated marked elevations in ATP, UTP, and GTP (Fig. 1). Serial measurements of three grey collies showed that during each cycle of neutropenia and neutrophilia these nucleotides varied regularly. As shown for grey collie 2980 (Fig. 2, left 

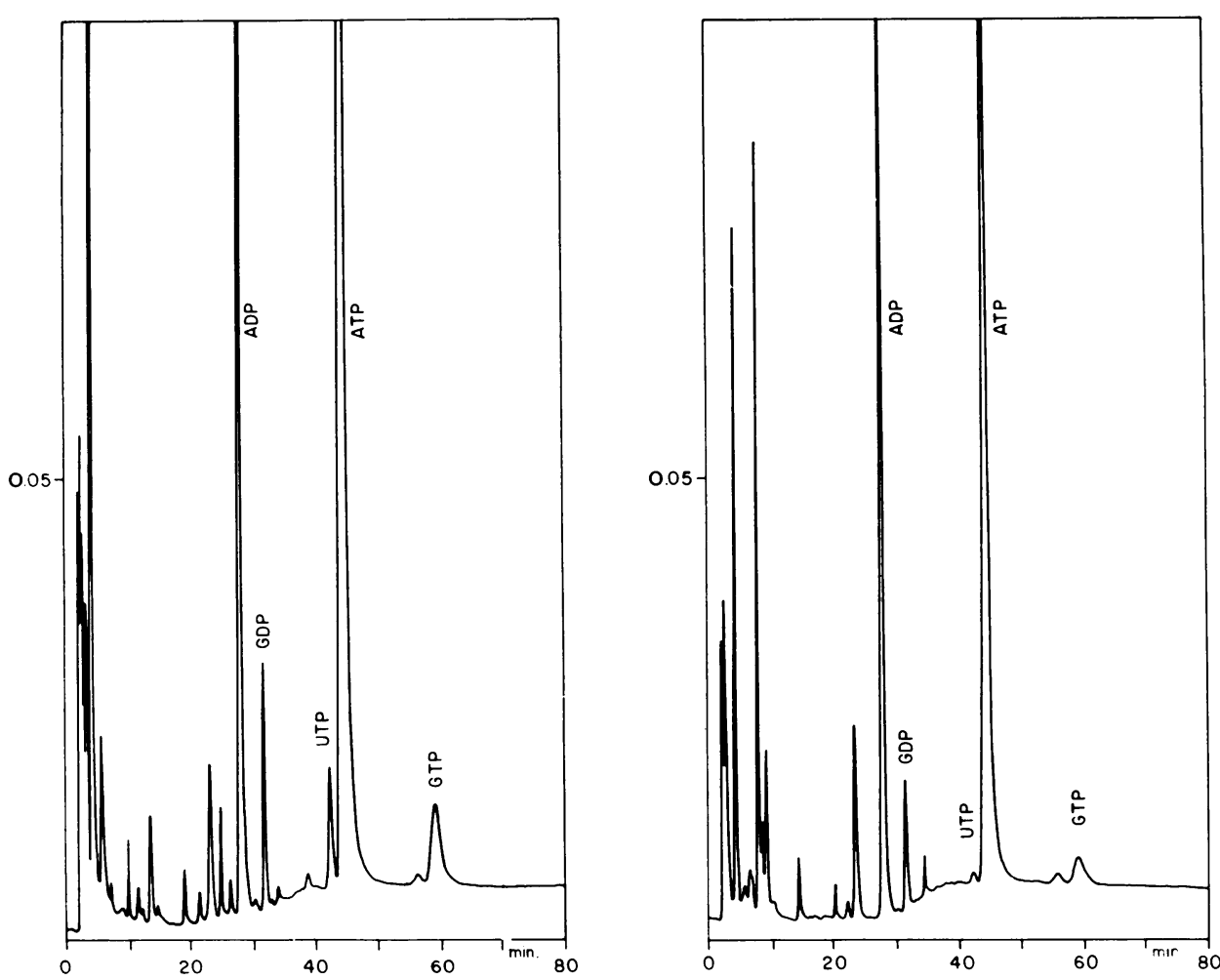

FIGURE 1 Nucleotide profiles of erythrocyte extracts from grey collie 2980 during a neutropenic period (left hand panel) and normal collie 678 (right hand panel). Extracts from $60 \mu \mathrm{l}$ of packed erythrocytes were analyzed.

panel), the nucleotide levels tended to rise during neutropenia and fall to normal levels as the neutrophil count recovered. Temporal changes in nucleotides of this magnitude were not observed in five normal dogs.

Nucleotides in cyclophosphamide-induced neutropenia. To demonstrate that the changes observed in the grey collies were not merely the consequence of neutropenia, we produced similar changes in neutrophil counts in three normal dogs with single doses of cyclophosphamide. The sequential levels of ATP, GTP, and PMN count following cyclophosphamide showed no apparent correlation between nucleotide levels and the fluctuation in neutrophil count (Fig. 2, right panel).

The sequential data from grey collie 2980 and the cyclophosphamide-treated normal dog 2939 were divided into the periods of neutropenia (PMN $<1,500$ / $\mu \mathrm{l})$ and neutrophilia $(\mathrm{PMN}>1,500 / \mu \mathrm{l})$, and the corresponding mean nucleotide values \pm SEM were compared (Table I). This procedure was useful because it gave a within dog comparison of nucleotides, and enabled each dog to serve as its own control. For the normal dog there was no significant increase in any of the erythrocyte nucleotide levels during the period of neutropenia. Similar results were obtained from two other normal dogs treated with cyclophosphamide. For the grey collie the time of neutropenia was highly sig-

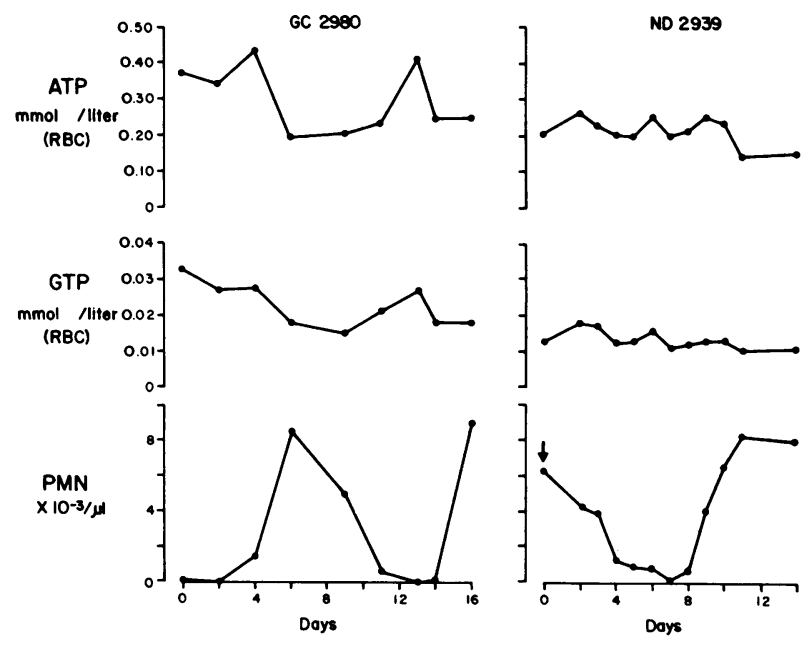

Figure 2 Plots of sequential neutrophil count, erythrocyte ATP and GTP for grey collie 2980 (left) and normal dog 2939 (right) after a single dose of cyclophosphamide, $20 \mathrm{mg} /$ $\mathrm{kg}$, administered on day shown by arrow. 
TABLE I

Erythrocyte Nucleotide Levels in a Normal Dog after Cyclophosphamide Treatment and a Grey Collie ${ }^{\circ}$

\begin{tabular}{|c|c|c|c|c|c|c|}
\hline \multirow[b]{2}{*}{ Nucleotide } & \multicolumn{2}{|c|}{ Grey collie 2980} & \multirow[b]{2}{*}{$P \downarrow$} & \multicolumn{2}{|c|}{ Normal dog $2939+$ cyclophosphamide } & \multirow[b]{2}{*}{$P \mathfrak{l}$} \\
\hline & $\mathrm{PMN}<1,500 / \mu \mathrm{l}$ & $\mathrm{PMN}>1,500 / \mu \mathrm{l}$ & & $\mathrm{PMN}<1,500 / \mu \mathrm{l}$ & $\mathrm{PMN}>1,500 / \mu \mathrm{l}$ & \\
\hline ADP & $0.314 \pm 0.0057$ & $0.308 \pm 0.0040$ & $>0.25$ & $0.166 \pm 0.0022$ & $0.164 \pm 0.0032$ & $>0.25$ \\
\hline GDP & $0.032 \pm 0.0011$ & $0.033 \pm 0.0008$ & $=0.05$ & $0.017 \pm 0.0007$ & $0.017 \pm 0.0006$ & $>0.5$ \\
\hline UTP & $0.013 \pm 0.0014$ & $0.008 \pm 0.0005$ & $=0.004$ & $0.006 \pm 0.0003$ & $0.006 \pm 0.0009$ & $>0.25$ \\
\hline ATP & $0.352 \pm 0.0299$ & $0.217 \pm 0.0111$ & $=0.002$ & $0.215 \pm 0.0075$ & $0.213 \pm 0.0198$ & $>0.5$ \\
\hline GTP & $0.025 \pm 0.0020$ & $0.017 \pm 0.0011$ & $=0.005$ & $0.012 \pm 0.0008$ & $0.014 \pm 0.0012$ & $=0.15$ \\
\hline Neutrophil count/ $\mu \mathrm{l}$ & $\begin{array}{r}543 \pm 259 \\
n=6 \S\end{array}$ & $\begin{array}{c}7,500 \pm 1,258 \\
n=3\end{array}$ & $<0.001$ & $\begin{array}{c}748 \pm 202 \\
n=5\end{array}$ & $\begin{array}{c}6,207 \pm 787 \\
n=7\end{array}$ & \\
\hline
\end{tabular}

- Values are means \pm SEM millimole/liter RBC.

$\ddagger$ For comparison within each animal's data between PMN $<1,500 / \mu \mathrm{l}$ and $\mathrm{PMN}>1,500 / \mu \mathrm{l}$.

$\S$ Number of observations.

nificantly correlated with increases in UTP, ATP, and GTP. There was no significant difference in mean levels of GDP and ADP during neutropenia and neutrophilia in the grey collie. The higher ADP and GDP levels in grey collie 2980 were a consistent observation in this dog, and were not observed in three other grey collies during hematopoietic cycling or in normal dogs, which all had nucleoside diphosphate levels similar to those recorded for ND 2939 (Table I). The cause of the elevated ADP and GDP in this one grey collie was not known. But whatever the level of nucleoside diphosphate, no significant within dog temporal variations were observed.

Nucleotides in lithium-treated normal and grey collie dogs. We measured the erythrocyte nucleotide

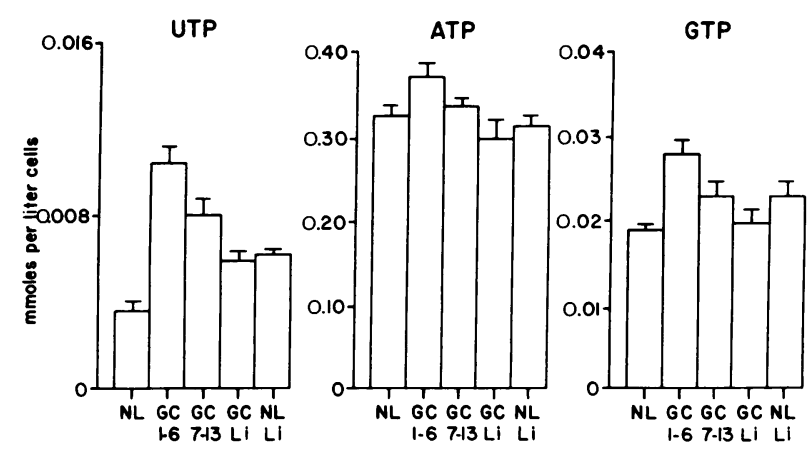

FIGURE 3 Erythrocyte ATP, GTP, and UTP levels (millimoles/liter RBC) for a series of experiments with: normal collies (NL), $n=10$; grey collies during days of neutropenia (GC 1-6), $n=11$; grey collies during days when neutrophil counts approached normal (GC 7-13), $n=12$; grey collies during lithium treatment (GC Li), $n=12$; and normal collies during lithium treatment (NL Li), $n=8$. The columns represent the mean nucleotide levels and bars are standard errors of the mean. $P$ values for all NL vs. GC $1-6$ comparisons were $<0.05$; for all $\mathrm{NL} \mathrm{Li}$ vs. GC $\mathrm{Li}$ were $>0.30$. levels in two normal dogs and two grey collies (GC 592 and GC 064) before and during the administration of lithium carbonate. For comparison, nucleotide levels for the grey collies were averaged over two periods, corresponding to neutropenia (days 1-6) and recovery (days 7-13). As shown above in a different dog, UTP, ATP, and GTP were elevated during neutropenia in these grey collies (Fig. 3). Lithium treatment of the grey collies normalized the levels of ATP and GTP and decreased uridine $5^{\prime}$-triphosphate (UTP) levels towards normal. For the lithium-treated grey collies and lithium-treated normal dogs, there were no significant differences in the nucleotide triphosphates.

Nucleotide levels in normal and phlebotomized dogs. The published values of ATP in normal dog erythrocytes (RBC) have been tabulated by Parker (30) and vary from 0.25 to $0.60 \mathrm{mmol} /$ liter $\mathrm{RBC}$ with a mean value of $0.46 \mathrm{mmol} /$ liter $\mathrm{RBC}$. We have measured erythrocyte ATP, GTP, and UTP in 11 normal dogs and found the following mean values \pm SEM and range: ATP, $0.331 \pm 0.018(0.413-0.214)$; GTP, $0.020 \pm 0.003(0.028-0.012)$; UTP, $0.005 \pm 0.002(0.015$ - 0.001) $\mathrm{mmol} /$ liter RBC.

Since the differences in erythrocyte nucleotide levels fluctuated in parallel with reticulocytes in the grey collies, variation in the proportion of reticulocytes could explain these differences. Over the hematopoietic cycle reticulocyte counts in grey collies vary between 0.2 and $3.5 \%$. A normal dog was bled to induce reticulocytosis and blood samples were collected and analyzed in triplicate. At a reticulocyte level of $1.1 \%$ the ATP, GTP, and UTP levels \pm SD were $0.395 \pm 0.015,0.024 \pm 0.004$, and $0.010 \pm 0.002 \mathrm{mmol} /$ liter $\mathrm{RBC}$, respectively, and at $6.2 \%$ reticulocytes the ATP, GTP, and UTP levels \pm SD were $0.327 \pm 0.025$, $0.023 \pm 0.002$, and $0.011 \pm 0.002 \mathrm{mmol} /$ liter $\mathrm{RBC}$, respectively. For the purines there was a decrease in 

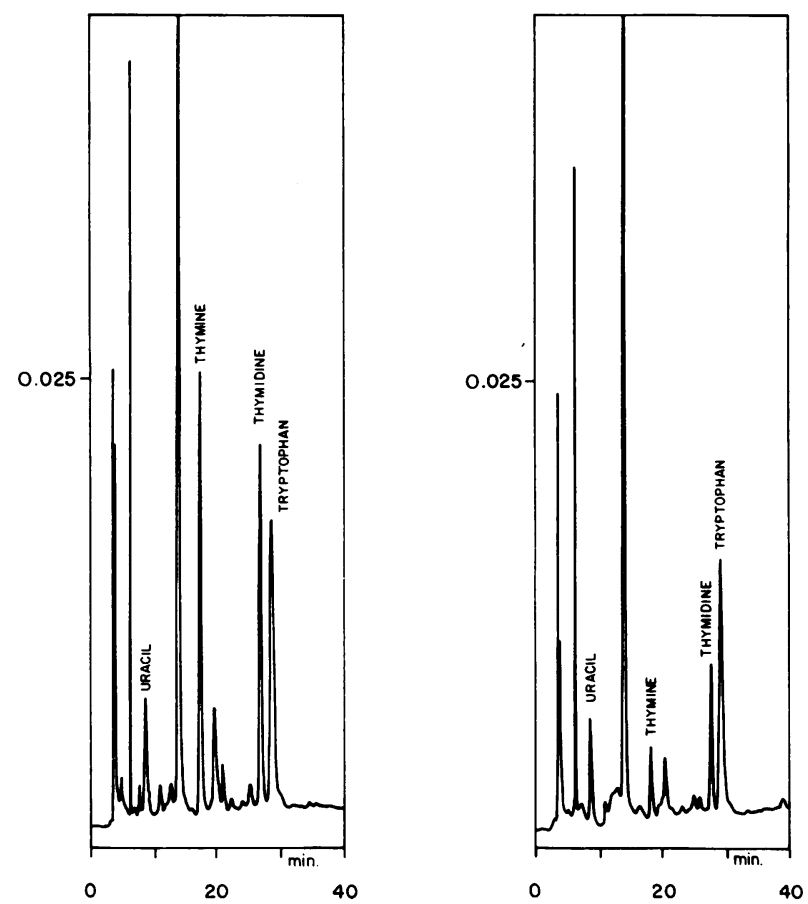

Figure 4 Chromatograms of plasma extracts from grey collie 2980 (left hand panel) and normal dog 2939 (right hand panel). The samples analyzed were derived from $160 \mu \mathrm{l}$ plasma.

level with increasing reticulocyte count, and for UTP there was an insignificant increase in level with increasing reticulocytes $(P>0.2)$.

Plasma nucleosides and bases in normal and grey collie dogs. In preliminary surveys of purine and pyrimidine nucleosides and bases in plasma from grey collies and normal dogs marked differences were seen in thymine and thymidine while uracil and the amino acid tryptophan did not differ (Fig. 4). A plot of sequential plasma thymine values and PMN counts for grey collie 2980 (not coincident with erythrocyte data shown above) and normal dog 2939 after a single cyclophosphamide dose is shown in Fig. 5. The grey collie showed a greater than twofold variation in thymine over the neutrophil cycle period, with the peak occurring at the time of neutropenia and falling towards normal values with neutrophil recovery. The normal dog treated with cyclophosphamide did not show thymine variation with the decrease of PMN count to zero or the increase to normal PMN number.

The plasma levels of thymine, thymidine, and uracil for grey collies 2980, and 592, and normal dog 2939 after cyclophosphamide treatment were divided into periods corresponding to neutropenia (PMN $<1,500$ / $\mu \mathrm{l})$ and neutrophilia $(\mathrm{PMN}>1,500 / \mu \mathrm{l})$. The mean values \pm SEM are shown in Table II with the $P$ values from their comparison. The normal dog showed no signif- icant increase in any of the pyrimidines with the fall in PMN number, whereas the grey collies displayed a significant increase in thymine during the time of neutropenia. The grey collies showed an increase in thymidine with neutropenia that is not statistically significant and, like the normal dog, showed no change in uracil level with neutrophil fluctuation.

For normal dog 3488 sampled over a 3-wk period $(n=6)$, the plasma values \pm SEM for thymine, thymidine, and uracil were $4.4 \pm 0.22,9.1 \pm 0.40$, and $4.7 \pm 0.25 \mu \mathrm{mol} /$ liter, respectively. The thymine and uracil levels are comparable with those recorded in Table II for normal dog 2939 following cyclophosphamide treatment and the thymidine level is similar to that recorded for the grey collies during neutrophilia.

Enzyme assays. We have measured erythrocyte and leukocyte (buffy coat) ADA and purine NP levels in grey collie 2980 and normal dog 3488 (Table III). The lack of ADA activity in dog erythrocytes has a precedent as the absence of other purine reutilization enzymes has been reported from the seal and eel (NP and hypoxanthine guanine phosphorylase transferase, HGPRT, respectively) (31). The erythrocyte NP level is $\sim 0.1 \%$ that in humans and complements the lack of ADA as the product of the ADA reaction provides the major substrate for NP. The leukocyte enzyme levels are in agreement with those reported in human leukocytes (32).

\section{DISCUSSION}

The serial measurement of erythrocyte purine and pyrmidine nucleotides in grey collie dogs showed a

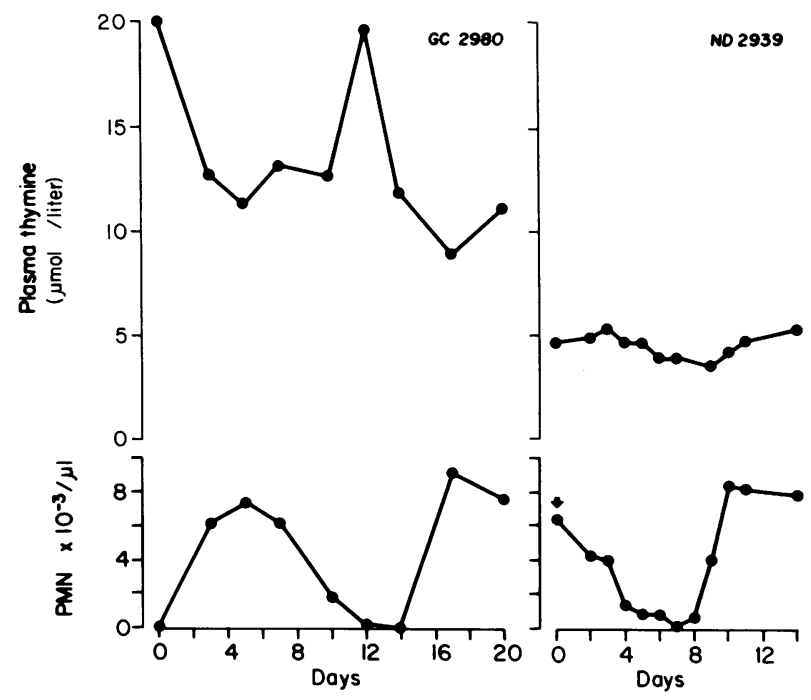

Figure 5 Plots of sequential neutrophil count and plasma thymine for grey collie 2980 and normal dog 2939 after a single dose of cyclophosphamide on day indicated by arrow. 
TABLE II

Plasma Uracil, Thymine, and Thymidine Concentrations in a Normal Dog After Cyclophosphamide Treatment and Two Grey Collies ${ }^{\circ}$

\begin{tabular}{|c|c|c|c|c|c|c|}
\hline \multirow[b]{2}{*}{ Pyrimidine } & \multicolumn{2}{|c|}{ Grey collies 2980 and 592} & \multirow[b]{2}{*}{$P \downarrow$} & \multicolumn{2}{|c|}{ Normal dog $2939+$ cyclophosphamide } & \multirow[b]{2}{*}{$P t$} \\
\hline & $\mathrm{PMN}<1,500 / \mu \mathrm{I}$ & $\mathrm{PMN}>1,500 / \mu \mathrm{l}$ & & $\mathrm{PMN}<1,500 / \mu \mathrm{l}$ & $\mathrm{PMN}>1,500 / \mu \mathrm{l}$ & \\
\hline Uracil & $4.98 \pm 0.263$ & $4.83 \pm 0.220$ & $>0.25$ & $3.98 \pm 0.260$ & $3.87 \pm 0.151$ & $>0.5$ \\
\hline Thymine & $19.70 \pm 1.683$ & $12.55 \pm 0.503$ & $<0.001$ & $4.40 \pm 0.164$ & $4.80 \pm 0.257$ & $>0.10$ \\
\hline Thymidine & $10.15 \pm 1.460$ & $8.05 \pm 1.072$ & $>0.15$ & $5.08 \pm 0.211$ & $5.89 \pm 0.315$ & $=0.03$ \\
\hline Neutrophil count $/ \mu \mathrm{l}$ & $\begin{array}{c}140 \pm 91 \\
n=6 \S\end{array}$ & $\begin{array}{c}8,558 \pm 2,074 \\
n=15\end{array}$ & $<0.001$ & $\begin{array}{r}748 \pm 203 \\
n=5\end{array}$ & $\begin{array}{c}6,207 \pm 787 \\
n=7\end{array}$ & $<0.001$ \\
\hline
\end{tabular}

- Values are means \pm SEM $\mu \mathrm{mol} /$ liter plasma.

† For comparison within each animal's data between PMN $<1,500 / \mu \mathrm{l}$ and PMN $>1,500 / \mu \mathrm{l}$.

$\S$ Number of observations.

perodic rise coincident with times of neutropenia and a decrease as neutrophil counts increased. In particular the triphosphates UTP, ATP, and GTP were significantly elevated during periods of neutropenia.

It was shown that the variations in nucleotides were not just a consequence of neutropenia by demonstrating the absence of any cycling effect in normal dogs made neutropenic with cyclophosphamide. In these dogs there were no significant variations in erythrocyte nucleotide levels with the decrease or increase of neutrophils. Dividing the data into times of neutropenia and relative neutrophil abundance and comparing each dog with itself gave a statistical comparison appropriate to periodic variation and also enabled us to use the dog under investigation as its own control, thus excluding between dog variation from our analysis.

The probability that nucleotide cycling was caused by variation in the number of reticulocytes was investigated by phlebotomy of normal dogs. There was no increase in erythrocyte ATP or GTP with a greater than fivefold increase in reticulocyte number. For UTP there was an increase in concentration with increased reticulocyte number, but not of a magnitude to ac-

TABLE III

Enzyme Activities for Grey Collie and Normal Dog

\begin{tabular}{|c|c|c|c|c|}
\hline & \multicolumn{2}{|c|}{ RBC: } & \multirow{2}{*}{\multicolumn{2}{|c|}{$\frac{\mathrm{WBC}}{\mu \mathrm{mol} / \mathrm{h} / \mathrm{mg} \text { protein }}$}} \\
\hline & \multirow{2}{*}{$\frac{\mu \mathrm{mol} / \mathrm{h} / \mathrm{g} \mathrm{Hb}}{\mathrm{ADA}}$} & \multirow{2}{*}{$\frac{\mu \mathrm{mol} / \mathrm{min} / \mathrm{g} \mathrm{Hb}}{\mathrm{NP}}$} & & \\
\hline & & & $\mathrm{ADA}^{\circ}$ & NP \\
\hline GC 2980 & ND & 0.21 & 0.72 & 2.55 \\
\hline ND 3488 & ND & 0.22 & 2.07 & 2.69 \\
\hline
\end{tabular}

ND, not detectable $<0.01 \mu \mathrm{mol} / \mathrm{h}$ per $\mathrm{g} \mathrm{Hb}$.

- Normal values for dogs not previously published. Range for ADA levels in human lymphocytes is $0.72-3.10 \mu \mathrm{mol} / \mathrm{h}$ per $\mathrm{mg}$ protein $(32,46)$. count for the changes in this pyrimidine nucleotide that we observed in the cycling grey collie. In grey collie dogs the reticulocytes increased about fivefold during each cycle reaching a peak value during the period of most severe neutropenia.

The virtual absence of adenosine deaminase and purine nucleoside phosphorylase from dog erythrocytes suggests that adenosine reutilization via these enzymes and hypoxanthine phosphoribosyl transferase to produce inosine 5 -monophosphate is not important, which is in contrast to human erythrocytes. Species differences in purine metabolism may also be reflected in the dissimilar ratios of ATP/ADP between dog and human erythrocytes. In dogs we have observed ATP/ ADP ratios of 1 to 2.5 , whereas this ratio in human erythrocytes is between 5 and 7 (24).

The effect of oral lithium treatment of grey collies was to lower the elevated levels of UTP, GTP, and ATP observed during neutropenia towards those present in grey collies with more normal neutrophil counts. The lithium-treated normal dogs and grey collies had similar erythrocyte nucleotide levels. This is probably not merely a consequence of the cessation of neutrophil cycling that results from lithium administration because neutrophil variation in itself does not cause changes in erythrocyte nucleotides as demonstrated by the cyclophosphamide treatment of normal dogs. The known effects of lithium are quite various and include enhanced mitosis of lymphocytes (33), modulation of adenylate cyclase (34), and increased granulocytopoiesis (35). The detailed mechanism by which lithium abrogates cycling in grey collies is not known.

The analysis of grey collie dog plasma showed abnormally high concentrations of thymidine and thymine, with the latter showing a peak level at the time of neutropenia. In general, thymidine is produced from the diet and DNA breakdown, and can be further degraded to thymine or reutilized to give deoxyribosylthymine monophosphate (dTMP) (36). The normal 
dogs made neutropenic with cyclophosphamide did not show elevations in plasma thymine or thymidine. This suggests that the increased catabolism of DNA following decline in neutrophil number was not the source of the excess thymine and thymidine in grey collie plasma. The metabolism of these pyrimidines in human leukocytes has been extensively reviewed (37, 38 ). An enzyme abnormality could be involved in the elevated plasma thymine and thymidine. The action of phosphatase or nucleotidase activity on dTMP will produce thymidine and could be involved in its accumulation in grey collie dog plasma. The activity of alkaline phosphatase in leukocytes has been studied and is inversely related to DNA synthesis $(39,40)$. Thymidine phosphorylase catalyzes the reversible interconversion of thymine and thymidine (36) and its activity is subject to modification by thymine (41). It has been suggested that under conditions where there has been a stimulus to DNA synthesis, thymidine phosphorylase could play a regulatory role in dTMP production $(38,42)$. In particular the control of thymidine phosphorylase may be dependent upon intracellular thymine concentrations (43). Furthermore, it has recently been shown that decreased activity of thymidine phosphorylase in human leukemic cells is associated with increased sensitivity to thymine-induced growth inhibition, and increased intracellular accumulation of deoxyribosylthymine triphosphate (dTTP) (44).

Whatever the source of the pyrimidines, their accumulation in grey collies could lead to the intracellular overproduction of dTTP, a known inhibitor of ribonucleotide reductase (23). Studies of adenosine deaminase and purine nucleoside phosphorylase-deficient patients has implicated ribonucleotide reductase inhibition as a major cause of their defective lymphopoiesis (20-22). Thus, inhibition of this enzyme in grey collies by an imbalance in the supply of TTP could account for their neutropenia.

Although the increased levels of purine and pyrimidine metabolites we have observed in grey collies may not seem compatible with a single enzyme defect, it is noteworthy that in human adenosine deaminase deficiency, besides elevations in adenine nucleotides, the levels of other purine and pyrimidine nucleotides were increased greater than twofold in the lymphocytes of an affected child (45). Also, purine nucleoside phosphorylase deficiency is associated with the large overproduction of purines (20-22).

These results have established a link between abnormal concentrations of purine and pyrimidine metabolites and cyclic hematopoiesis in grey collie dogs and enable us to hypothesize that this metabolic derangement is primarily responsible for the defective neutrophil proliferation characteristic of this disorder.
These findings suggest that studies of these purine and pyrimidine abnormalities should lead to the biochemical defect and a pathogenetic mechanism to explain hematopoietic cycling in this unusual disease.

\section{ACKNOWLEDGMENTS}

We wish to thank Dr. C. Ronald Scott for his interest and support, Clinton Cleveland and Elin Rodger for their technical expertise, and Annett Allred for typing skills.

This work was supported by U. S. Public Health Service grants GM 15253 and AM 18951-06. Presented in part at the 23rd Annual Meeting of the American Society of Hematology, San Antonio, TX, December 1981.

\section{REFERENCES}

1. Lund, J. E., G. A. Padgett, and R. L. Ott. 1967. Cyclic neutropenia in grey collie dogs. Blood. 29: 452-461.

2. Dale, D. C., S. B. Ward, H. R. Kimball, and S. M. Wolff. 1972. Studies of neutrophil production and turnover in grey collie dogs with cyclic neutropenia. J. Clin. Invest. 51: $2190-2196$.

3. Dale, D. C., D. W. Alling, and S. M. Wolff. 1972. Cyclic hematopoiesis: the mechanism of cyclic neutropenia in grey collie dogs. J. Clin. Invest. 51: 2197-2204.

4. Patt, H. M., J. E. Lund, and M. A. Maloney. 1973. Cyclic hematopoiesis in grey collie dogs: a stem-cell problem. Blood. 42: 873-884.

5. Adamson, J. W., D. C. Dale, and R. J. Elin. 1974. Hematopoiesis in the grey collie dog. Studies of the regulation of erythropoiesis. J. Clin. Invest. 54: 965-973.

6. Lange, R. D., J. B. Jones, C. Chambers, Y. Quinn, and J. C. Sparks. 1976. Erythropoiesis and erythrocytic survival in dogs with cyclic hematopoiesis. Am. J. Vet. Res. 37: 331-334.

7. Dunn, C. D. R., J. B. Jones, J. D. Jolly, and R. D. Lange. 1977. Progenitor cells in canine cyclic hematopoiesis. Blood. 50: 1111-1120.

8. Dunn, C. D. R., J. D. Jolly, J. B. Jones, and R. D. Lange. 1978. Erythroid colony formation in vitro from the marrow of dogs with cyclic hematopoiesis: interrelationship of progenitor cells. Exp. Hematol. 6: 701-708.

9. Dunn, C. D. R., J. B. Jones, J. D. Jolly, and R. D. Lange. 1978. Cell proliferation of canine cyclic hematopoietic marrow in diffusion chambers. Proc. Soc. Exp. Biol. Med. 158: 50-53.

10. Hammond, W. P., E. R. Engelking, and D. C. Dale. 1979. Cyclic hematopoiesis: effects of endotoxin on colony-forming cells and colony-stimulating activity in grey collie dogs. J. Clin. Invest. 63: 785-792.

11. Hammond, W. P., J. W. Adamson, and D. C. Dale. 1982. Canine cyclic haematopoiesis: the effect of endotoxin on erythropoiesis. Br. J. Haematol. 50: 283-294.

12. Dale, D. C., and R. G. Graw, Jr. 1974. Transplantation of allogeneic bone marrow in canine cyclic neutropenia. Science (Wash. DC). 183: 83-84.

13. Weiden, P. L., B. Robinett, T. C. Graham, J. Adamson, and R. Storb. 1974. Canine cyclic neutropenia. A stem cell defect. J. Clin. Invest. 53: 950-953.

14. Jones, J. B., T. J. Yang, J. B. Dale, and R. D. Lange. 1975. Canine cyclic hematopoiesis: marrow transplantation between littermates. Br. J. Haematol. 30: 215223. 
15. Hammond, W. P., and D. C. Dale. 1980. Lithium therapy of canine cyclic hematopoiesis. Blood. 55: 26-28.

16. Hammond, W. P., and D. C. Dale. 1982. Cyclic hematopoiesis: effects of lithium on colony-forming cells and colony-stimulating activity in grey collie dogs. Blood. 59: 179-184.

17. Giblett, E. R., J. E. Anderson, F. Cohen, B. Pollara, and H. J. Meuwissen. 1972. Adenosine deaminase deficiency in two patients with severely impaired cellular immunity. Lancet. II: 1067-1069, 1972.

18. Giblett, E. R., A. J. Ammann, D. W. Wara, R. Sandman, and L. K. Diamond. 1975. Nucleoside phosphorylase deficiency in a child with severely defective T-cell immunity and normal B-cell immunity. Lancet. I: 10101013.

19. Pollara, B., H. J. Meuwissen, R. J. Pickering, and I. H. Porter. 1979. Inborn Errors of Specific Immunity. Academic Press, Inc., New York.

20. Giblett, E. R., S.-H. Chen, and W. R. A. Osborne. 1981 Inherited defects in purine salvage enzymes associated with immunodeficiency states. In Contemporary $\mathrm{He}$ matology and Oncology. R. Silber, A. S. Gordon, J. LoBue, and F. M. Muggia, editors. 2: 263-286.

21. Martin, D. W., and E. W. Gelfand. 1981. Biochemistry of diseases of immunodevelopment. Annu. Rev. Biochem. 50: $845-877$.

22. Kredich, N. M., and M. S. Hershfield. 1983. In The Metabolic Basis of Inherited Disease. 5th edition. J. B. Stanbury, J. B. Wyngaarden, D. S. Fredrickson, J. L. Goldstein, and M. S. Brown, editors. McGraw-Hill, New York. 1157-1183.

23. Thelander, L., and P. Reichard. 1979. Reduction of ribonucleotides. Annu. Rev. Biochem. 48: 133-158.

24. Beutler, E. 1975. Red cell metabolism: a manual of biochemical methods. Grune \& Stratton, Inc., New York.

25. Garrett, C., and D. V. Santi. 1979. A rapid sensitive high pressure liquid chromatography assay for deoxyribonucleotide triphosphates in cell extracts. Anal. Biochem. 99: 268-275.

26. Hartwick, R. A., and P. R. Brown. 1975. The performance of microparticle chemically-bonded anion exchange resins in the analysis of nucleotides. J. Chromatogr. 112: 651-662.

27. Hartwick, R. A., and P. R. Brown. 1976. Evaluation of microparticle chemically bonded reversed-phase packings in the high-pressure liquid chromatographic analysis of nucleosides and their bases. Anal. Biochem. 126: 679-691.

28. Osborne, W. R. A., and N. Spencer. 1973. Partial purification and properties of the common inherited forms of adenosine deaminase from human erythrocytes. Biochem. J. 133: 117-123.

29. Osborne, W. R. A. 1980. Human red cell purine nucleoside phosphorylase: purification by biospecific affinity chromatography and physical properties. J. Biol. Chem. 255: 7089-7092.

30. Parker, J. C. 1977. Solute and water transport in dog and cat red blood cells. In Membrane Transport in Red
Cells. J. C. Ellory and V. L. Lew, editors. Academic Press, Ltd., London. 427-465.

31. Parks, R. E., P. R. Brown, Y.-C. Cheng, K. C. Agarwall, C. M. Kong, R. P. Agarwal, and C. C. Parks. 1973. Purine metabolism in primitive erythrocytes. Comp. Biochem. Physiol. 45B: 355-365.

32. Sullivan, J. L., W. R. A. Osborne, and R. J. Wedgwood. 1977. Adenosine deaminase activity in lymphocytes. $\mathrm{Br}$. J. Haematol. 17: 157-158.

33. Shenkman, L., W. Borkowsky, and B. Shopsin. 1980. Lithium as an immunologic adjuvant. Med. Hypoth. 6: $1-6$.

34. Gelfand, E. W., H-M. Dosch, D. Hastings, and A. Shore. 1979. Lithium: a modulator of cyclic AMP-dependent events in lymphocytes? Science (Wash. DC). 203: 365367.

35. Rossof, A. H., and W. A. Robinson. 1980. Lithium effects on granulopoiesis and immune function. Plenum Publishing Corp., New York.

36. Henderson, J. F., and A. R. P. Paterson. 1973. Nucleotide Metabolism. Academic Press, Inc., New York.

37. Gallo, R. C. 1971. Synthesis and metabolism of DNA and DNA precursors by human normal and leukemic leukocytes. Acta Haematol. 45: 136-158.

38. Perry, S., and R. C. Gallo. 1970. Physiology of human leukemic leukocytes: kinetic and biochemical considerations. In Regulation of Hematopoiesis. S. A. Gordon, editor. Appleton, New York. 1221-1271.

39. Trubowitz, S., D. Feldman, S. W. Morgenstern, and V. M. Hunt. 1961. The isolation, purification and some properties of the alkaline phosphatase of human leukocytes. Biochem. J. 80: 369-374.

40. Fernley, H. N. 1971. Mammalian alkaline phosphatases. In The Enzymes. IV. P. Boyer, editor. Academic Press, Inc., New York. 417-447.

41. Gallo, R. C., and S. Perry. 1969. The enzymatic mechanisms for deoxythymidine synthesis in human leukocytes. IV. Comparisons between normal and leukemic leukocytes. J. Clin. Invest. 48: 105-116.

42. Gallo, R. C., and T. R. Breitman. 1968. The enzymatic mechanisms for deoxythymidine synthesis in human leukocytes. III. Inhibition of deoxythymidine phosphorylase by purines. J. Biol. Chem. 243: 4943-4951.

43. Gallo, R. C., S. Perry, and T. R. Breitman. 1967. The enzymatic mechanisms for deoxythymidine synthesis in human leukocytes. I. Substrate inhibition by thymine and activation by phosphate or arsenate. J. Biol. Chem. 242: 5059-5068.

44. Fox, R. M., S. K. Piddington, E. H. Tripp, N. P. Dudman, M. H. N. Tattersall. 1979. Thymidine sensitivity of cultured leukemic lymphocytes. Lancet. I: 391-393.

45. Schmalstieg, F. C., J. A. Nelson, G. C. Mills, T. M. Monahan, A. S. Goldman, and R. M. Goldblum. 1977. Increased purine nucleotides in adenosine deaminase-deficient lymphocytes. J. Pediatr. 91: 48-51.

46. Tung, R., R. Silber, F. Quagliata, M. Conklyn, J. Gottesman, and R. Hirschhorn. 1976. Adenosine deaminase activity in chronic lymphocytic leukemia. J. Clin. Invest. 57: 756-761. 